\title{
The One-Stage Premaxillary Repositioning and Bone Graft Alveoloplasty in Bilateral Alveolar Cleft: A Preliminary Study
}

\author{
AHMED A.K. MOUSA, M.D.*; SALAH M. MAKLAD, M.D.** and MOHAMAD A. ELKHOLY, M.Sc.*** \\ The Departments of Plastic \& Reconstructive Surgery*, Faculty of Medicine, Aswan University, Radiology** and Dentistry***, \\ Aswan University Hospitals, Aswan, Egypt
}

\begin{abstract}
This prospective clinical study is designed to present seven consecutive cases with bilateral cleft lip and palate at age of mixed dentition to be managed by simultaneous premaxilla repositioning and bone grafting of their clefts at period between 2014 and 2019 in Plastic Surgery Department, Aswan University. Clinical and cephalometric radiography assessment during follow-up period up to two years' post-operation to evaluate premaxilla position, stability, anterior palatal growth. Indications, contraindications and the pre- and post-operative orthodontic role technique were defined and proved to be a safe procedure. In properly selected patients, it can produce early one-step stable maxillary arch with good aesthetic result. No relapse or major complication were detected.
\end{abstract}

Background: The bilateral alveolar cleft associated with markedly prominent premaxillary segment always possessed a surgical challenge because of the paucity of local flaps for watertight closure and high bone graft alveoloplasty failure rate. The orthodontic workup alone to mold unrestricted growing premaxillary segment could not optimize its position with the unfavorable functional and aesthetic outcome. In addition, the repeated surgical intervention may addon patient suffering and affect maxillary growth rate.

Objective: Evaluation of the premaxillary viability, position, bone graft stability, and early growth after simultaneous premaxillary repositioning and alveolar bone grafting inalveolar cleft patients.

Patients and Methods: Seven patients aged between 6 and 11 years were subjected to perioperative orthodontic workups to optimize the position of the premaxilla for its surgical correction before bone grafting. Then, the simultaneous premaxillary repositioning and bone graft alveoloplasty technique was done. Surgical indications, contraindications with orthodontic role were evaluated and discussed.

Results: This prospective clinical study presents the outcome of seven consecutive pediatric patients who complained ofalveolar clefts with severe premaxillary segment protrusion. It was conducted during the period between 2014 and 2020 in the Plastic Surgery Department, Aswan University Hospitals. The results were assessed by clinical examination and cephalometric tracing for at least two years for evaluating the premaxillary position, functional outcome, upper jaw stability, and early growth affection.

Conclusion: This technique proved to be a safe procedure in appropriately selected patients. It can produce an early stable maxillary arch with good aesthetic results besides less surgical interventions. No relapse or significant complications were documented.

Clinical Relevance The treatment of wide bilateral cleft alveolus patients is still based on experience and expert opinions. This preliminary study deals with markedly protruded premaxilla with bilateral alveolar cleft by combined orthodontic and one-stage premaxillary repositioning with bone graft alveoloplasty. An extended study period assessment for midfacial and maxillary growth is necessary.

Key Words: Bilateral wide alveolar cleft - Premaxilla repositioning osteotomy - Orthodontic.

Conflict of Interest: No potential conflict of interest relevant to this article was reported.

\section{INTRODUCTION}

Canine eruption needs solidified bony ground, which is provided by a bone graft. Therefore, it isagreed that the appropriate age for bone graftalveoloplasty is during the mixed dentition period where canine root reaches about half of its length $[1,2]$. It is not uncommon to see severe forward and vertical over development of the premaxilla. This may make conventional alveolar bone grafting difficult or even not possible without complications [3]. Proper management techniques and sequence of the wide alveolar cleft with severe premaxillary segment protrusion are debatable inliterature $[3,4]$. In the past, total excision of the premaxilla has been described $[\mathbf{5 , 6 ]}$. Here, the rise of concern of severe nasomaxillary growth retardation after the premaxillary setback at an early age was questioned many years ago and made many cleft surgeons not preferring such a technique. Therefore, Monroe and coworkers suggested that vomerine osteotomy should avoid-theoretically-injury of epiphyseal line growth center at vomero-premaxillary suture [7].

This study aimed to present our experience in treating bilateral alveolar cleft patients with severe upward and anterior premaxillary segment protrusionby simultaneous premaxillary repositioning 
and bone grafting. Surgical indications, contraindications with orthodontic role were discussed.

\section{PATIENTS AND METHODS}

\section{Patients selection:}

The data of 47 cases with alveolar and postalveolar cleft who were candidates foriliac bone marrow grafting at the Aswan Plastic Surgery Department during 2014 and 2019 were primarily analyzed. Through 28 unilateral and 19 bilateral, only seven patients, five males and two females, aged 6 to 11, were selected to be managed by onestage premaxillary impaction after osteotomy with bilateral bone graft alveoloplasty. The indications for this selection included severe overgrowth protrusion of the premaxilla in all patients with added lateral premaxillary segment rotation in two of them (Fig. 1).

Orthodontic workup: It started at least one year before surgical intervention. In four cases, palatal expansion was performed using a customized Vshaped palatal expander appliance hinged on screws at posterior molars. The remaining three cases were not subjected to palatal expansion as it was not indicated. The orthodontist advised stopping palatal expansion once the adequate width was achieved and avoid overexpansion. This should give proper space to position the impacted premaxillary segment during surgery. Any interfering primary teeth at cleft sides were removed, preferably at 4 to 6 weeks before the surgery, to facilitate proper flap closure.

\section{Surgical technique:}

The procedure was done for all cases under general anesthesia and prophylactic antibiotic. A surgical incision was done along the lateral cleft sides and proceeded upward to the alveolar cleft.
The incision was done at the sides of the premaxilla without extensive undermining. On one side only, the incision extended posteriorlyto the vomerine attachment with premaxillae to be gently osteotomized $5 \mathrm{~mm}$ back to premaxillary-vomerine suture lineby a 4-mm sharp rhinoplasty osteotome (Figs. 2A,B). The upper premaxillary attachment was gently released from the septum of the nose. Then, the segment was impacted in an upward and backward direction in all cases. Flaps were dissected off the inner side of the palatal mucoperiosteum and from the premaxilla to ensure "Watertight" closure of the nasal side of the cleft and avoid dehiscence and graft loss. The already created space between the cleft and inner (nasal side) layer was impacted with the cancellous iliac bone. A buccal flap layer was turned over the impacted bone graft to complete gingival (oral) layer closure. At the end of the surgery, a dental splint with \# 22-gauge wire was secured onto the upper alveolus to stabilize the repositioned premaxilla and prevent its relapse. The patients were instructed to be on soft diet for eight weeks with frequent oral antiseptic solution for propermouth hygiene.

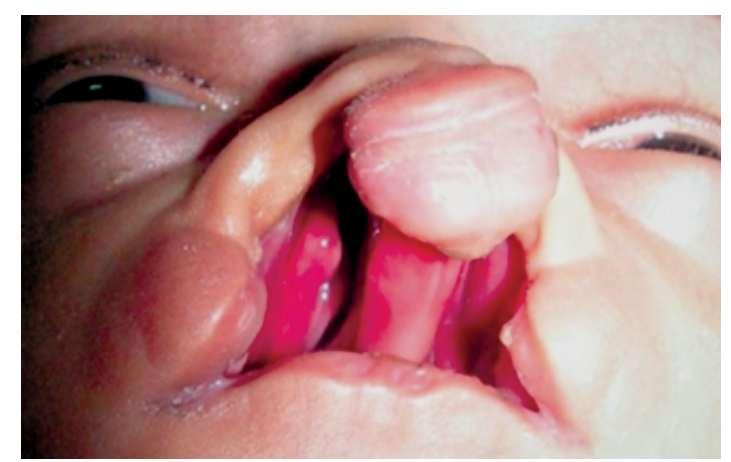

Fig. (1): A male child at onemonth age showed severe premaxillary segment protrusion with deviation to left side associated with wide bilateral alveolar and palatal clefts. At the age of 7 years, as shown in Fig. (6-A), he was operated upon for alveolar cleft by our study technique.

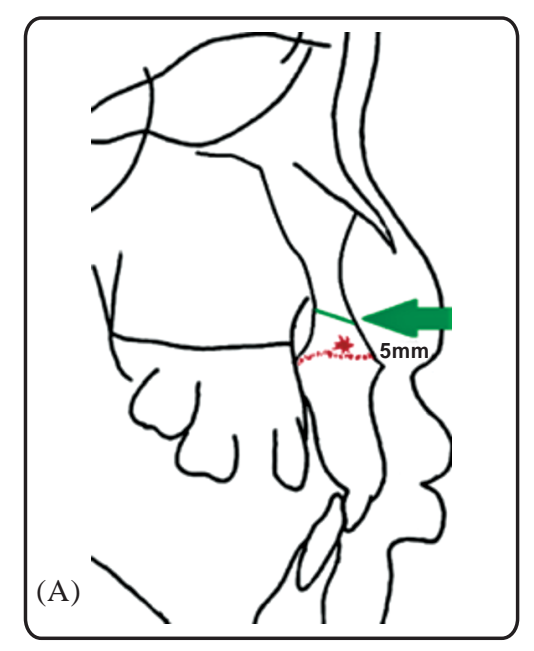

(B)

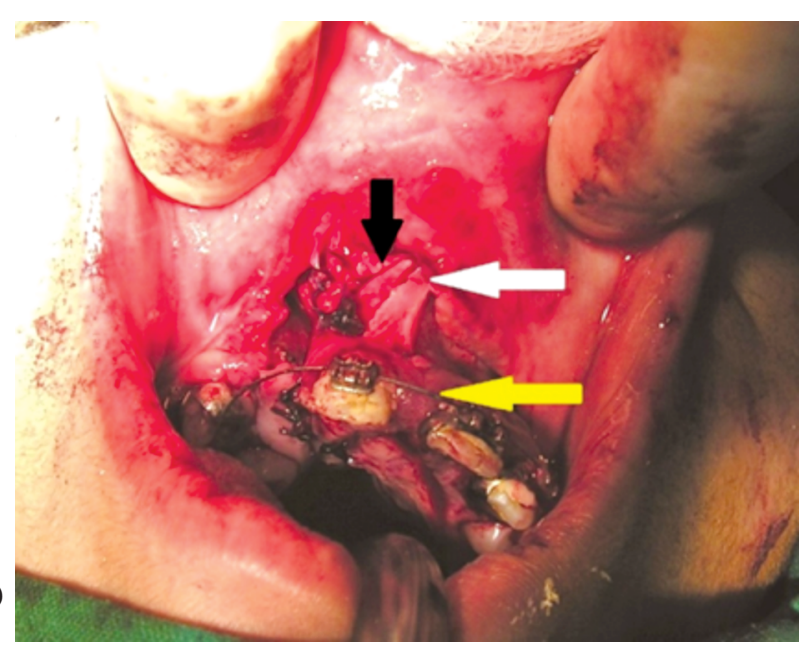

Fig. (2): (A) Illustrated diagram shows the site of premaxilla-vomerine suture (red dots) and hypothesized growth center (red star). The green arrow points to the osteotomy site $(5 \mathrm{~mm}$ above and behind the suture line). (B) A photo of dissected right side premaxilla mucosa to denude the cleft up to premaxilla-vomerine suture (White arrow). Black arrow points at exposed septum where osteotomy to be done. Yellow arrow points at archwire hold on teeth secured to maintain impacted premaxilla stable in place for eight weeks. 


\section{Management evaluation:}

Follow-up meetings with the surgeon and orthodontist were regular. We removed the metal splint after ten weeks of surgery and replaced it with the orthodontic archwire. The assessment included premaxillary segment viability, stability, incisor inclination, segment relapse, bone graft success, and any complications. This follow-up extended up to two years for each case. Posteroanterior and lateral cephalometry (Fig. 3A,B) measurement tracing with statistical analysis were done for the cooperative children at preoperative period; T1 and 18 months after the surgery; T2. Here, we considered cephalometric reference measures for each pediatric age $[\mathbf{8 , 9}$. In the cephalograms (Fig. 4A,B), the $\mathrm{X}$-axis represented a horizontal reference line, and $\mathrm{Y}$-axis was the perpendicular line on it. Both lines were used as reference lines to assess premaxilla position and growth direction. The progress of $\mathrm{T} 1$ to $\mathrm{T} 2$ was illustrated with the "analysis of variance" and was compared for the significant difference by "paired $t$-test". The vertical reduction of the palatal plane of the premaxilla was plotted against the height of the vomeronasal septal complex with a " $t$-test." Examining the premaxillary angular bending on the vomeronasal septal complex was assessed by tracing reference points at the vertical axis of S1 to S4 in posteroanterior (PA) view. Each patient's T1 and T2 images were superimposed with actual relative dimensions were designed by the computer office program into a single superimposed figures for comparison (Figs. 5A,B). T1 and T2 were analyzed by using the "paired $t$-test" for all tests $(p<0.05)$.
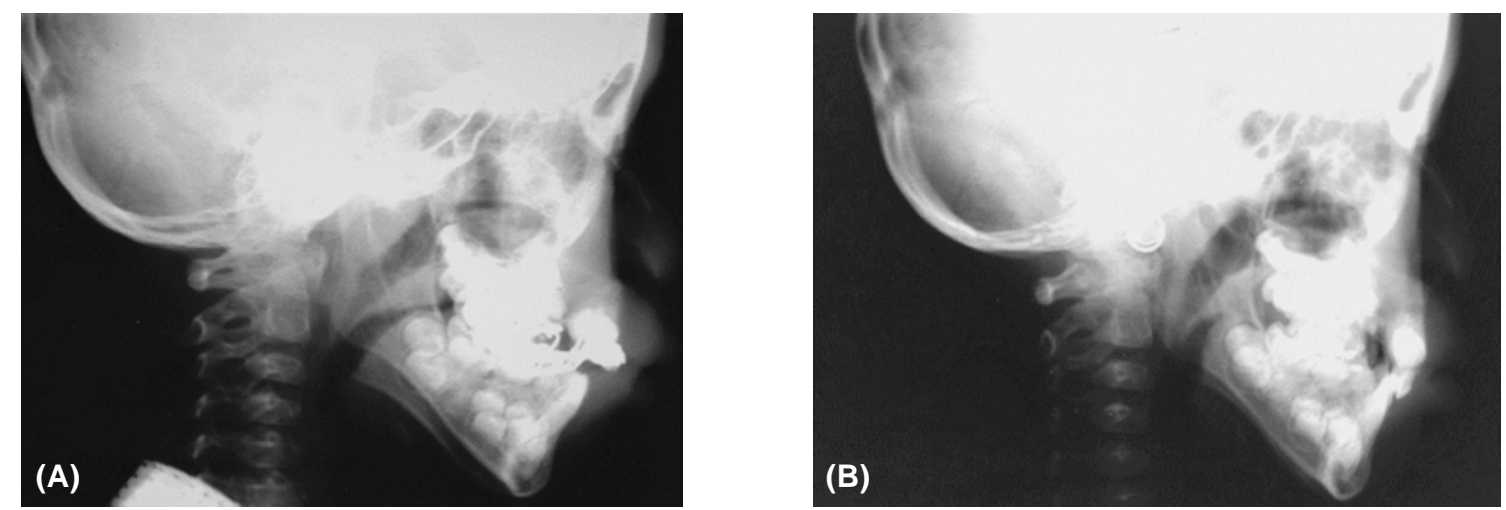

Fig. (3): Lateral Cephalometry of patient No. 2: (A) Pre-operative orthodontic management achieved after palatal expansion. (B) The same patent eighteen months post-operative. Note improved premaxillary position with vertical shortening and stable premaxillary impaction. The incisor overbite increased.
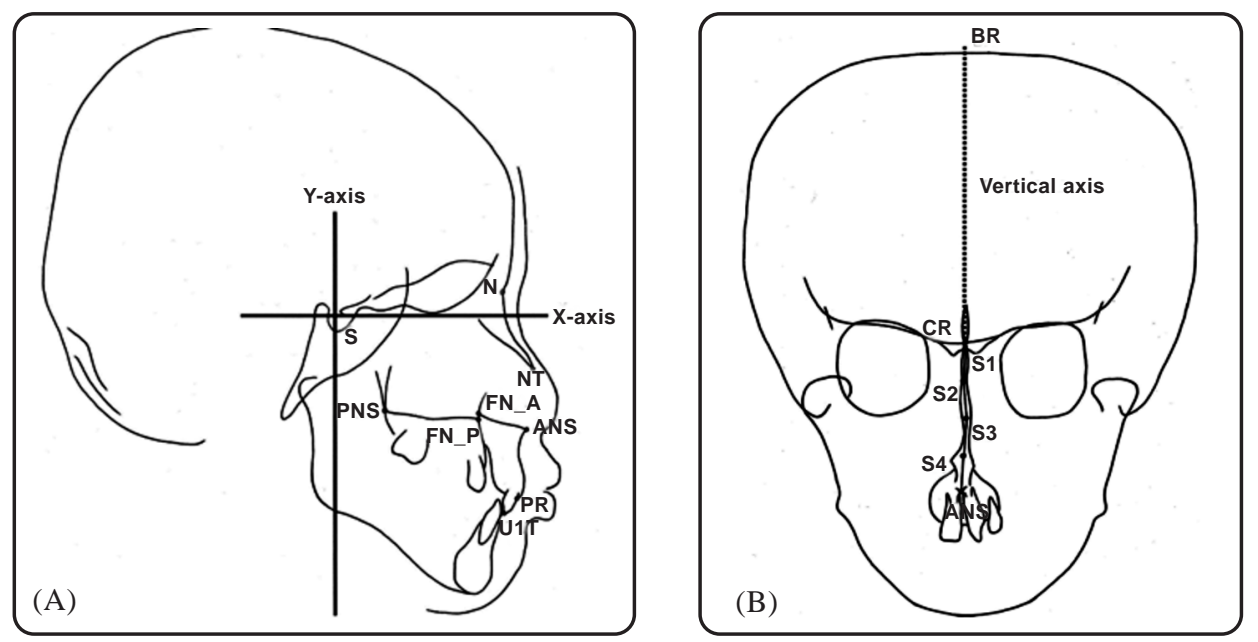

Fig. (4): (A) The Lateral view Cephalometry reference landmarks \& lines.S=Sella. N=Nasion. FN=Frontonasal point. PR=Prosthion. $\mathrm{N}=$ Nasion. $\mathrm{U} 1 \mathrm{~T}=$ tip of maxillary incisor. $\mathrm{A}=$ Point anterior premaxilla. $\mathrm{P}=\mathrm{Posterior}$ palatal point. ANS=Anterior nasal spine. PNS=Posterior nasal spine. The X-axis has constructed horizontally down anteriorly to the Sella-Nasion line by 7 degrees. Y-axis; The vertical reference line upon $\mathrm{X}$-axis. $(\mathrm{FN}$ to $\mathrm{P})=$ The line intersects the frontonasal process of the maxillary bone and nasal side of the palatal plane. $(\mathrm{FN}-\mathrm{A})=$ The outline of the premaxillary palatal plane intersects a point of the nasal side \& frontonasal process of the maxilla. $(\mathrm{FN}-\mathrm{A} \& \mathrm{PNS})=$ Plane of the palate at buccal segments. (ANS-FN) = premaxillary plane palate. $(\mathrm{ANS}-\mathrm{PR})=$ Premaxillary dentoalveolar height. (B)Posteroanterior Cephalometry: The reference line here is the vertical line from the base of (CR); Crista Gali to the (BR); Bregma in the skull. It is divided into an equal four distances, S1 to S4, representing landmark points from the anterior nasal spine (ANS) to (CR). [with permission from Rakosi, Thomas". An atlas and manual of cephalometric radiography" -1982] [9]. 
Fig. (5): (A) The superimposed $\mathrm{T} 1$ and $\mathrm{T} 2$ cephalometric tracing drawings for Case No. 2 (same in Fig. 5) shows the preoperative solid line cephalometry and dotted line at 18 month's follow-up post-operative. Premaxillary segment movement with the palatal plane and occlusal adjustment. (B) Superimposed T1 \& T2 cephalometric trace for Case No. 3. Notice reduced premaxilla-nasal complex with adjustment of lower jaw incisors after 18 months of growth. The palatal plane was aligned, and the mandible showed vertical and horizontal growth with proper occlusion.
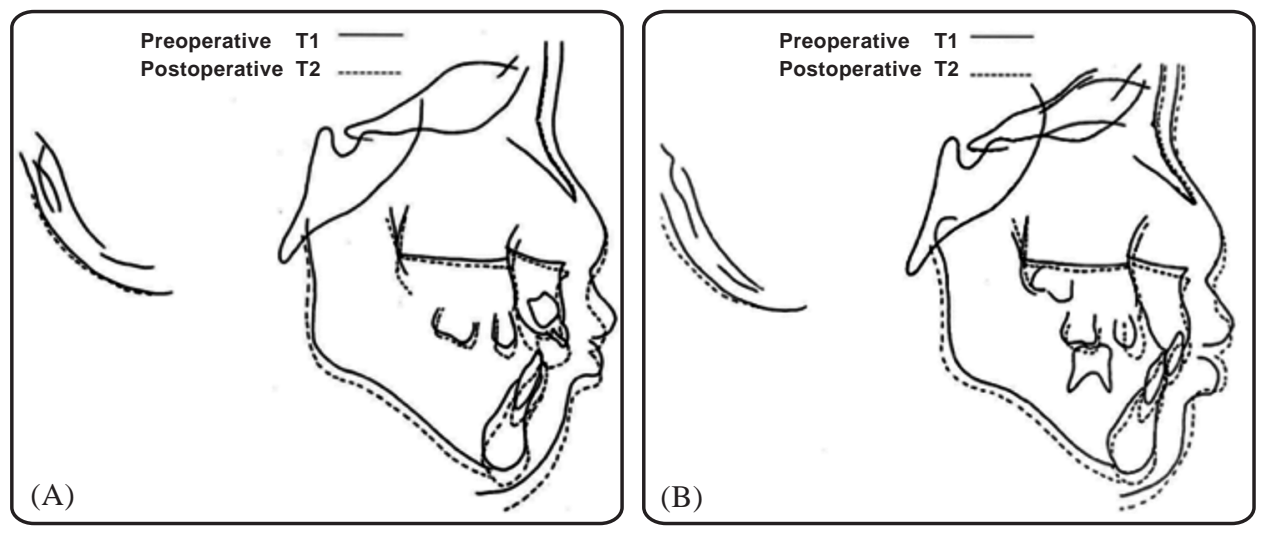

\section{RESULTS}

All seven patients tolerated the procedure and the post-operative period went uneventfully except for one case with mild wound infection at one side. Table (1) shows each case's age/gender, radiologic cleft width, premaxillary surgical movement, and stability after one year. The average intraoperative width between the premaxilla and lateral maxillary segments was improved to be $16.2 \mathrm{~mm}$ on average by orthodontic work up before surgical intervention. After surgery, the overbite was significantly improved by $2 \mathrm{~mm}$ on average, with the significant intrusion of the premaxillary palatal plane for about $6.5 \mathrm{~mm}$. (Fig. 6A,B). The anterior vomeronasal septum unit was significantly reduced at vertical direction without bending in an average of $2.7 \mathrm{~mm}$. The rotational adjustment of the premaxilla segment

(A)

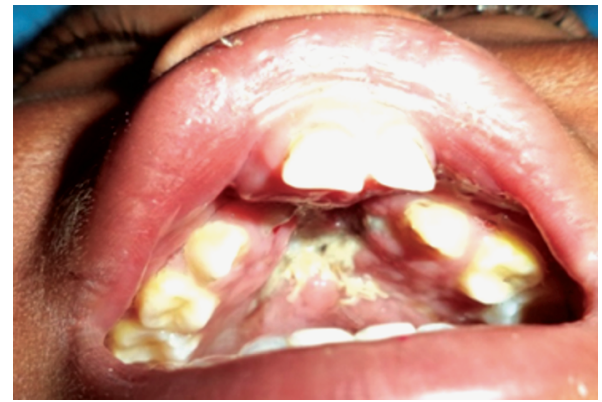

was an average of $2.6 \mathrm{~mm}$. The occlusal planes of the buccal and premaxillary segments were almost at the same level with no vascular compromise or ischemic loss of the premaxilla in all patients. Completeclinical bone stabilization was seen in 6 cases after two years. One patient with a postsurgical infection in one side, with a recurrent small oronasal fistula after ten days, showed minor bone consolidation at 12 weeks, with a mild premaxillary instability. Here, the bone support on the fistula side, as appeared in the radiograph, was less than the uncomplicated side. This oronasal fistula was repaired in layers after six months without further bone grafting. All patients showed acceptable alignment of the dental arch and aesthetic outcome (Fig. 7A-C). The follow-up period lasted two years ( +8 months for three cases).

(B)

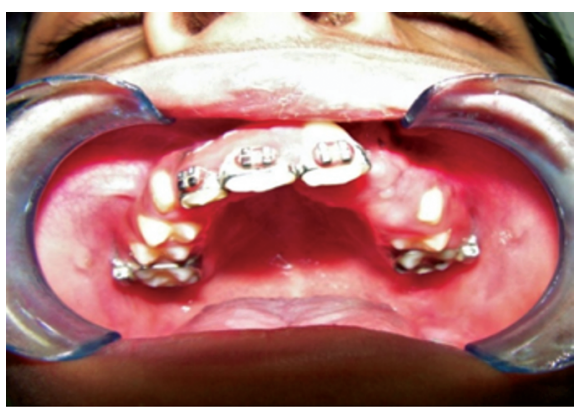

Fig. (6): (A) View during orthodontic workup and before surgical intervention (6 months): Illustrating the reduced premaxillary segment to be partially aligned with maxillary arch, the oversized and vertically overdeveloped premaxilla, and the presence of the unrepaired alveolar clefts. (B) Postoperative view of the maxillary arch after six months of alveoloplasty. The continuity of the alveolar ridge has been restored with healed cleft sides by a solidified bone.

(A)

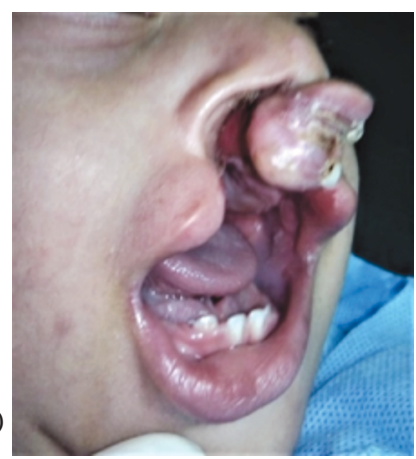

(B)

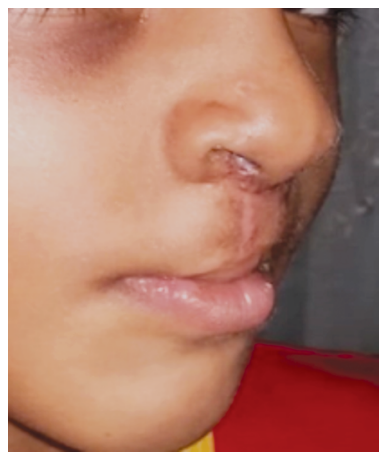

(C)

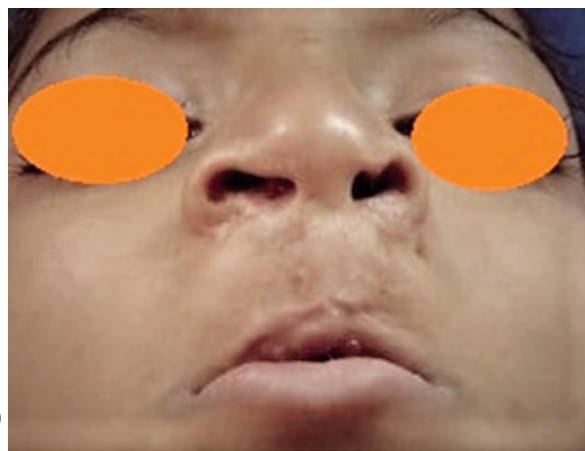

Fig. (7): Cases No. 5: (A) At the age of 4 months. (B) Oblique lateral view at the age of 8 years (The timing of the surgery was at the age of 6). (C) Worm-view with accepted premaxillary reduction and aesthetic appearance. 
Table (1): Movement of premaxilla at surgical procedure and its stability after one year.

\begin{tabular}{clcccc}
\hline Case no. & Age/Sex & Backward $(\mathrm{mm})$ & Vertical $(\mathrm{mm})$ & Lateral $(\mathrm{mm})$ & Stability \\
\hline 1 & $8 / \mathrm{M}$ & 5 & 3 & 0 & No \\
2 & $11 / \mathrm{m}$ & 7 & 2 & 4 to right & No \\
3 & $9 / \mathrm{F}$ & 4 & 2 & 3 to left & No \\
4 & $8 / \mathrm{M}$ & 8 & 4 & 5 to right & Moderate \\
5 & $6 / \mathrm{F}$ & 9 & 3 & 3 to right & No \\
6 & $7 / \mathrm{M}$ & 5 & 2 & 0 & No \\
7 & $12 / \mathrm{M}$ & 8 & 3 & 3 to left & No \\
Average & 8.5 years & 6.5 & 2.7 & 2.6 & \\
\hline
\end{tabular}

\section{DISCUSSION}

In patients with bilateral alveolar clefts, thepremaxilla shows unrestricted growth at anterior and downward directions starting as early as the $10^{\text {th }}$ gestational period, with sometimes lateral rotation of the premaxillary segment due to loss of lip attachment effect. The patient has apparentanterior malocclusion with a wide oronasal fistula [5]. Consequently, this complicates for disruption of average oromaxillary balance and reflects on feeding, speech, and aesthetic appearance with psychological concerns $[6,7,8]$.

Marked protrusion with vertical overgrowth of the premaxilla represents a significant surgical challenge for surgical and orthodontic management [8]. Bardach [10] defined width of $1.5 \mathrm{~cm}$ as indicative of a wide anterior cleft palate and alveolus. Bumsted [11] defined an extremely wide alveolar cleft to be present in cases where "the width of the cleft defect is larger than the sum of the combined widths of the remaining palatal flap". There is no available agreed definition for wide alveolar cleft except that cleft with insufficient local flap for no tension closure $[\mathbf{8 , 1 0}]$. As advocated in the 1960s in the European and Scandinavian studies, the primary excision of the premaxilla is not used anymore by most centers. It has a devastating maxillary growth effect [11]. On the other hand, the two stages of bone graft alveoloplasty then corrective osteotomy of the premaxillary process in the second one became more popular in selected cases with accepted results [7]. Later on, by performingboth procedures simultaneously, surgeons are encouraged to close a sizeable oronasal fistula [12-16].

This study has a relatively short follow-up period to complete midfacial growth assessment, with small number of patients and a significant standard deviation. However, it showed promising preliminary results for simultaneous correction ofpremaxillary deformity and alveolar cleft with minor complications. This could be explained from three basic approaches: Surgical mobilization of the premaxilla, shortening of premaxillary dentoalveolar height, and pre-operative/post-operative orthodontic workup. Collaboration of the surgical and orthodontic decision and work gave the best functional and aesthetic outcome interest for the patient. This was achieved after backward impaction of the premaxilla and vertical dentoalveolar height reduction without significant vomeronasal complex lateral/medial angular deviation. Although post-operative overbite was moderately compromised due to dental intrusion and premaxillary incisor overeruption, no significant functional difference could be elicited.

Orthodontic upper alveolus adjustment has a critical role in restoring and preserving proper maxillary edge alignment at pre and post-operative periods [17]. Before the premaxillary corrective osteotomy, we had toensure adequate palatal expansion in four cases to maintain alignment of the maxillary arch with impacted premaxilla. The rapid expansion was easily achieved at this early age before maxillary bone fusion. The remaining three cases did not need palatal expansion as they had no lateral maxillary segments collapse. Typically, maxillary incisors of bilateral alveolar cleft patients exhibit an increased longitudinal axis bending with downward and/or lateral deviation of the premaxilla [18-20]. Here, changing the upper incisors' root direction perpendicular to the palatal plane during the surgery greatly facilitated upcoming orthodontic management and allowed easy incisor alignment. Immediate facial appearance improvement with minorgingival show deformity added for its benefit.

Patient selection for this technique is essential to expect the best functional and aesthetic outcome and avoid unnecessary premaxillary osteotomy. We could demonstrate some indications for simultaneous alveolar bone grafting and surgical premaxillary impaction. The first was the severe protrusion of the premaxilla. The technique al- 
lowed "watertight" closure of the oral and nasal mucosae without suture stress failure. This would not be very easy with the usual technique. The second was vertical over development to restore the maxillary alveolar continuity and restoring an early normal facial appearance. The third indication is severe lateral bending of the unrestricted premaxillary segment that is difficult to manage without corrective mobilization. The fourth was the closure of large oro-nasal fistulas, which significantly decreased the bone gap to help successful bone graft uptake with a favorable prognosis. The fifth, later decision of orthognathic; Lefort I osteotomy in cases with class three malocclusion would be easier in consolidated-one-unit upper jaw than non-united segments. Here we should confirm that surgical timing is an essential factor before severe palatal collapse and/or maxillary segment deformity.

It is contraindicated to do premaxillary osteotomy repositioning in some circumstances as inan early age before development of secondary dentition of canine root at the permanent maxilla, under challenging circumstances to achieve orthodontic expansion of the lateral segments, inadequate dental eruption, significantly underdeveloped premaxilla, and in patients whocould not have orthodontic management facilities. Itisalso contraindicated when previous surgical interventions at the area of lining mucosa, which may compromise vascularity of the premaxillary segment, or when it is difficult to properly fix the post-operative splint. That is why the pre-operative orthodontic assessment is preferred to be a routine workup before any cleft surgery. Patient and his/her parents' compliance to keep up with dental splint for eight to ten weeks is essential. Early upper jaw stability provides less time for dental splinting and prevents expected bone relapse [21,22]. Although invasiveness of the technique, the advantage of no second surgical session for bone grafting was beneficial.

We should mention that this study is a preliminary assessment as the regard growth rate of the upper jaw. There is no apparent growth retardation within the follow-up period, although they are still under observation. A wide-scale project to provide further assessment of midfacial growth and occlusal status is needed.

\section{Conclusion:}

Although simultaneous one stage-surgery ofpremaxillary segment impaction and alveolar cleft bone grafting was technically demanding, it showed effective early stabilization of the upper jaw. It is a single-stage invasive procedure with the accepted aesthetic and functional outcome if done with careful anatomical dissection not to harm the vomeronasal suture. When the patient was appropriately being selected, this technique solves the problem of the severely displaced premaxilla after achieving a maxillary arch expansion. In addition, it both, solidifies the alveolar arch at an early age and reconstructs the oronasal fistula. Surgical, orthodontic, radiologic, and patients' parents collaboration in such cases is paramount. It is essential to follow-up same cases and add bigger numbers for further years to document upper jaw growth rate and effect on midface appearance before suggesting this technique as a standard for practice.

\section{REFERENCES}

1- Abyholm F.E., Bergland O. and Semb G.: Secondary bone grafting of alveolar clefts. A surgical/orthodontic treatment enabling a non-prosthodontic rehabilitation in cleft lip and palate patients. Scand. J. Plast. Reconstr. Surg., 15 (2): 127-140, 1981.

2- Bergland O., Semb G. and Abyholm F.E.: Elimination of the residual alveolar cleft by secondary bone grafting and subsequent orthodontic treatment. Cleft Palate J., 23 (3): 175-205, 1986.

3- Freihofer H.P., van Damme P.A. and Kuijpers-Jagtman A.M.: Early secondary osteotomy stabilization of the premaxilla in bilateral clefts. J. Craniomaxillofac. Surg., 19 (1): 2-6, 1991.

4- Pérez González, Araceli M.D.*; Arámburo-García and Rigoberto M.D.: Anatomical Reconstruction in Bilateral Cleft Lip with Mendoza Technique, Journal of Craniofacial Surgery, Vol. 29 (6): 1452-1456, 2018.

5- Latham R.A.: Development and structure of the premaxillary deformity in bilateral cleft lip and palate. Br. J. Plast. Surg., 26 (1): 1-11, 1973.

6- Motohashi N. and Pruzansky S.: Long-term effects of premaxillary excision in patients with complete bilateral cleft lips and palates. Cleft Palate J., 18 (3): 177-187, 1981.

7- Monroe C.W., Griffith B.H., McKinney P., Rosenstein S.W. and Jacobson B.N.: The surgical recession of the premaxilla and its effect on maxillary growth in patients with bilateral clefts. Cleft Palate J., 7: 784-793, 1970.

8- Latham R.A., Deaton T.G. and Calabrese C.T.: A question of the role of the vomer in the growth of the premaxillary segment. Cleft Palate J., 12: 351-355, 1975.

9- Rakosi and Thomas: An atlas and manual of cephalometric radiography; Thomas Rakosi; translated by R.E.K. Meuss Wolfe London, Vol. (1): 41-45, 1982.

10- Eldeeb M.E., Hinrichs J.E., Waite D.E., Bandt C.L. and Bevis R.: Repair of alveolar cleft defects with autogenous bone grafting: Periodontal evaluation. Cleft Palate J., 23 (2): 126-136, 1986.

11- Bardach J.: Book Salyer and Bardach's atlas of craniofacial and cleft surgery. Vol. II: Cleft lip and palate surgery. Philadelphia: Lippincott-Raven, 1999. 
12- Bumsted R.M.: A new method for achieving complete two-layer closure of a massive palatal cleft. Arch. Otolaryngol., 108: 147-50, 1982.

13- Eppley B.L., Sclaroff A. and Delfino J.J.: Secondary management of the premaxilla in bilateral cleft lip and palate patients. Journal of Oral \& Maxillofacial Surgery, 44 (12): 987-998, 1986

14- Robertson N.R. and Jolleys A.: Effects of early bone grafting in the complete clefts of lip and palate. Plastic \& Reconstructive Surgery, 42 (5): 414-421, 1968.

15- Lino M., Sasaki T., Kochi S., Fukuda M., Takahashi T. and Yamaguchi T.: Surgical repositioning of the premaxilla in combination with two-stage alveolar bone grafting in bilateral cleft lip and palate. Cleft Palate and Craniofacial Journal, 35 (4): 304-309, 1998.

16- Hayward J.R.: Management of the premaxilla in bilateral clefts. Journal of Oral \& Maxillofacial Surgery, 41 (8): 518-524, 1983.

17- Aburezq H., Daskalogiannakis J. and Forrest C.: Management of the prominent premaxilla in bilateral cleft lip and palate. Cleft Palate Craniofac J., 43 (1): 92-5, 2006.
18- Kobayashi S., Hirakawa T., Fukawa T., Satake T. and Maegawa J.: Synchronous Premaxillary Osteotomy with Primary Cheiloplasty for BCLP Patients with Protrusion of the Premaxillae. Plast. Reconstr. Surg. Glob. Open., 5 (11): e1402, 2017.

19- Lorenzoni D.C., Janson G., Bastos J.C., et al.: Evaluation of secondary alveolar bone grafting outcomes performed after the canine eruption incomplete unilateral cleft lip and palate. Clin. Oral Investig., 21 (1): 267-273, 2017.

20- Heidbuchel K.L., Kuijpers-Jagtman A.M. and Freihofer H.P.: An orthodontic and cephalometric study on the results of the combined surgical-orthodontic approach of the protruded premaxilla in bilateral clefts. J. Craniomaxillofac. Surg., 21 (2): 60-66, 1993.

22- Yilmaz, Hanife Nuray D.D.S., Ph.D.; Karabiber, Gulden D.D.S. and Erverdi, Nejat D.D.S., Ph.D.\$: A Novel Approach for the Reconstruction of Premaxilla by Archwise Distraction in Bilateral Cleft Lip and Palate, Journal of Craniofacial Surgery, Volume 30 (1): p e40-e43, 2019.

22- Emodi, Omri DMD; Shoshani-Dror, Dana DMD, M.Sc.; et al.: Osteotomy of the Premaxilla in Bilateral Cleft Lip, Journal of Craniofacial Surgery, 2021. 